\title{
High resolution radio study of the pulsar wind nebula within the supernova remnant G0.9+0.1
}

\author{
G. Dubner ${ }^{1}$, E. Giacani ${ }^{1}$, and A. Decourchelle ${ }^{2}$ \\ 1 Instituto de Astronomía y Física del Espacio (IAFE), CC 67, Suc. 28, 1428 Buenos Aires, Argentina \\ e-mail: [gdubner; egiacani]@iafe.uba.ar \\ 2 Service d'Astrophysique, Orme des Merisiers, CE-Saclay, 91191 Gif-sur-Yvette Cedex, France \\ e-mail: anne.decourchelle@cea.fr
}

Received 16 April 2008 / Accepted 13 June 2008

\begin{abstract}
Aims. We have conducted a study in radio wavelengths and in X-rays of the pulsar wind nebula (PWN) in the supernova remnant (SNR) G0.9+0.1 with the goal of investigating in detail its morphology and to accurately determine its characteristic parameters. Methods. To carry out this research we have observed the PWN at $\lambda 3.6$ and $6 \mathrm{~cm}$ using the Australia Telescope Compact Array (ATCA) and combined these data with existing multiconfiguration VLA data and single dish observations in order to recover information at all spatial scales. We have also reprocessed VLA archival data at $\lambda 20 \mathrm{~cm}$. From all these observational data we have produced high-fidelity images at the three radio frequencies with angular resolution better than $3^{\prime \prime}$. The radio data were compared to X-ray data obtained with Chandra and in two different observing runs with XMM-Newton.

Results. The new observations revealed that the morphology and symmetry suggested by Chandra observations (torus and jet-like features) are basically preserved in the radio range in spite of the rich structure observed in the radio emission of this PWN, including several arcs, bright knots, extensions and filaments. The reprocessed X-ray images show for the first time that the X-ray plasma fills almost the same volume as the radio PWN. Notably the X-ray maximum does not coincide with the radio maximum and the neutron star candidate CXOU J174722.8-280915 lies within a small depression in the radio emission. From the new radio data we have refined the flux density estimates, obtaining $S_{\text {PWN }} \sim 1.57 \mathrm{Jy}$, almost constant between $\lambda 3.6$ and $\lambda 20 \mathrm{~cm}$. For the whole SNR (compact core and shell), a flux density $S_{20 \mathrm{~cm}}=11.5 \mathrm{Jy}$ was estimated. Based on the new and the existing $\lambda 90 \mathrm{~cm}$ flux density estimates, we derived a spectral index $\alpha_{\mathrm{PWN}}=-0.18 \pm 0.04$ and $\alpha_{\text {shell }}=-0.68 \pm 0.07$. From the combination of the radio data with X-ray data, a spectral break is found near $v \sim 2.4 \times 10^{12} \mathrm{~Hz}$. The total radio PWN luminosity is $L_{\text {radio }}=1.2 \times 10^{35} \mathrm{erg} \mathrm{s}^{-1}$ when a distance of $8.5 \mathrm{kpc}$ is adopted. By assuming equipartition between particle and magnetic energies, we estimate a nebular magnetic field $B=56 \mu \mathrm{G}$. The associated particle energy turns out to be $U_{\text {part }}=5 \times 10^{47} \mathrm{erg}$ and the magnetic energy $U_{\text {mag }}=2 \times 10^{47}$ erg. The high ratio between magnetic and particles flux energy density suggests that the pulsar wind just started to become particle dominated. Based on an empirical relation between X-ray luminosity and pulsar energy loss rate, and the comparison with the calculated total energy, a lower limit of $1100 \mathrm{yr}$ is derived for the age of this PWN.
\end{abstract}

Key words. ISM: supernova remnants - X-rays: ISM - radio continuum: ISM - ISM: individual objects: G0.9+0.1

\section{Introduction}

Radio composite supernova remnants (SNRs) consist of a shell and a spectrally distinct inner nebula, presumably a pulsar wind nebula (PWN), powered by the wind of relativistic electron/positron pairs from a central pulsar. Only in a few cases, however, has the central pulsar been detected (see Kaspi \& Helfand 2002, for a review).

Recent observations of several composite SNRs carried out with Chandra X-ray Observatory have resolved out on arcsec scales complex structures in the interior of several PWNe. These structures include toroids, axial bipolar jets, wisps, etc. (Weisskopf et al. 2000; Helfand et al. 2001; Roberts et al. 2003, etc.). Images in the different spectral domains are essential to understanding the physics of PWNe. Particularly, the radio emission depends on the history of the nebula and represents the combination of the efficiency of the pulsar in providing accelerated particles and magnetic fields, and the expansion history. The expansion history, in turn, depends on the density and geometry of the medium that confines the relativistic particles and fields (i.e. the interior of the SNR, that includes stellar ejecta and the presence of forward and reverse shocks). The detailed analysis of the geometry and structure of the PWN and the parent SNR, can shed light on the coupling mechanisms between the neutron star, the relativistic wind nebula and the surrounding SNR plasma.

G0.9+0.1 $\left(\mathrm{RA}=17^{\mathrm{h}} 47^{\mathrm{m}} 21^{\mathrm{s}}, \mathrm{Dec}=-28^{\circ} 09^{\prime}, \mathrm{J} 2000\right)$ is a composite SNR located in the direction of the Galactic center and at about the same distance (assumed through this paper to be $8.5 \mathrm{kpc}$ ). It is characterized by a bright, centrally condensed synchrotron nebula, approximately $2^{\prime}$ in size, and a weak surrounding radio shell, about $8^{\prime}$ in size, for which radio spectral indices $\alpha_{\text {core }} \sim-0.12$ and $\alpha_{\text {shell }} \sim-0.6$ (where $S \propto v^{\alpha}$ ), have been proposed for the core and shell respectively (Helfand \& Becker 1987; La Rosa et al. 2000).

In the X-rays domain, the first detection was reported by Helfand \& Becker (1987) based on IPC-Einstein observations, who concluded that the observed flux could come either from the compact core or from a combination of core and part of the bright limb of the shell of G0.9+0.1. The core X-ray emission was detected by Mereghetti et al. (1998) using BeppoSAX satellite. Sidoli et al. (2000) later confirmed these results on the basis of better quality data. These early detections are indicative of the 
presence of a young neutron star powering the nebula, although no coherent pulsations are found.

Gaensler et al. (2001) presented the results of $35 \mathrm{ks}$ ACIS Chandra observations of the PWN, between 0.5 and $8.0 \mathrm{keV}$. From these images, the authors identify a faint semicircular arc and a jet-like feature that define a symmetry axis, which they interpret as evidence of a torus of emission in the pulsar's equatorial plane and a jet directed along the pulsar spin axis. No X-ray emission is detected in correspondence with the radio-shell nor its interior. Based on these observations the authors propose that the hard point-like X-ray source CXOU J174722.8-280915 detected at energies above $3 \mathrm{keV}$, is the best candidate for a central pulsar that would be powering the inner nebula. This point source has a rather low ratio of magnetospheric pulsar emission to surrounding nebular emission, with a luminosity that amounts to only $0.5 \%$ of the total PWN luminosity in the energy range 2-10 keV.

Porquet et al. (2003) carried out an X-ray study of the PWN within G0.9+0.1 using the XMM-Newton EPIC-MOS and EPIC-PN cameras. The images obtained in the energy band 1.5-12.0 keV show an amorphous nebula with a bright maximum towards the east surrounded by extended diffuse emission. At the spatial resolution of XMM-Newton ( $\left.8^{\prime \prime}\right)$, the arc and jet-like features noticed by Gaensler et al. (2001) are not obvious. The X-ray spectrum within the PWN softens from the core to the outskirts, consistent with synchrotron radiation losses of high energy electrons as they diffuse through the nebula. The $X M M-N e w t o n$ study also reveals spectral variations across the "arc-like feature" identified by Gaensler et al. (2001), with the eastern part of the arc having clear indications of a very hard photon index $(\Gamma \sim 1.0)$, opposite to the western part with a very soft spectrum $(\Gamma \sim 3.2)$.

Aharonian et al. (2005) reported the detection, for the first time, of gamma-ray emission in the direction of G0.9+0.1 at energies greater than $100 \mathrm{GeV}$ at a level of significance of $13 \sigma$. The very high energy gamma-rays, discovered using the HESS instrument, appear to originate in the pulsar wind nebula. The photon spectrum is compatible with a power law with photon index $\Gamma=2.4$.

In radio wavelengths, G0.9+0.1 is prominent at $57.5 \mathrm{MHz}$ and $80 \mathrm{MHz}$ (La Rosa \& Kassim 1985). It has also been observed at $843 \mathrm{MHz}$ (Gray 1994), at $330 \mathrm{MHz}$ as a part of the highresolution imaging of the Galactic Center region (Nord et al. 2004) (Fig. 1), and at $1.5 \mathrm{GHz}$ and $4.8 \mathrm{GHz}$ (Helfand \& Becker 1987).

This paper attempts to analyze the morphology and spectral properties of the PWN in G0.9+0.1 in the radio range, based on new high-resolution radio images obtained from observations at $4.8 \mathrm{GHz}(\lambda 6 \mathrm{~cm})$ and at $8.4 \mathrm{GHz}(\lambda 3.6 \mathrm{~cm})$ carried out with the Australia Telescope Compact Array ${ }^{1}$ and from reprocessed archival $\mathrm{VLA}^{2}$ data at $1.4 \mathrm{GHz}(\lambda 20 \mathrm{~cm})$. Also the X-ray emission associated with this nebula has been re-analyzed including new XMM-Newton data and reprocessed Chandra observations.

\footnotetext{
1 The Australia Telescope Compact Array (/Parkes telescope/Mopra telescope/Long Baseline Array) is part of the Australia Telescope which is funded by the Commonwealth of Australia for operation as a National Facility managed by CSIRO.

2 The VLA of the National Radio Astronomy Observatory is a facility of the NSF operated under cooperative agreement by the Associated Universities Inc.
}

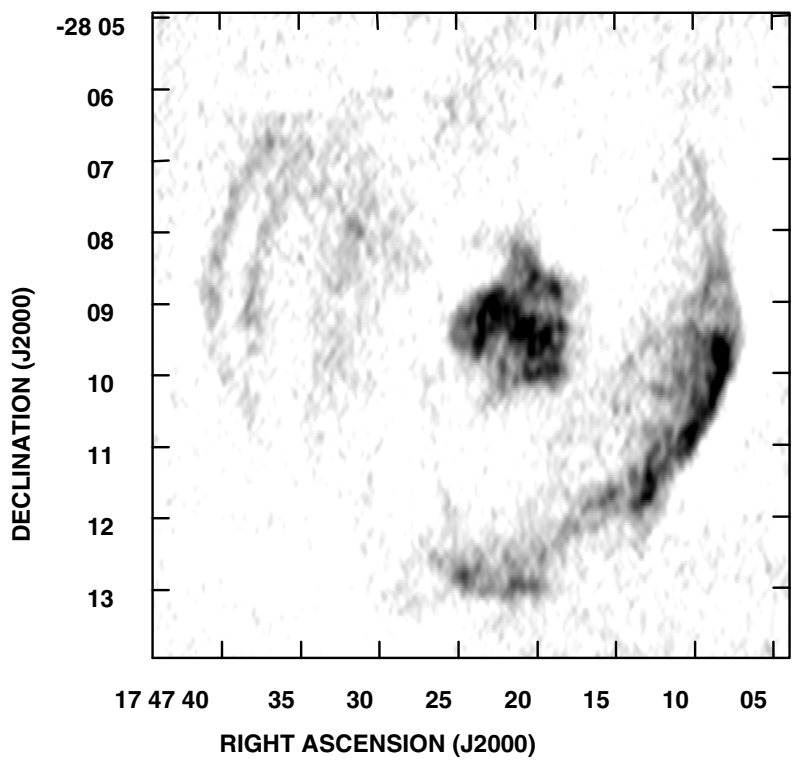

Fig. 1. The SNR G0.9+0.1 at $330 \mathrm{MHz}$ as taken from Nord et al. (2004).

\section{Observations}

\subsection{Radio data at $3.6 \mathrm{~cm}, 6 \mathrm{~cm}$ and $20 \mathrm{~cm}$}

The radio continuum emission of G0.9+0.1 was simultaneously observed at $\lambda 6 \mathrm{~cm}$ and $3.6 \mathrm{~cm}$ using the Australia Telescope Compact Array (ATCA) during 12 h on 15/16 January 2004. The array was used in the $6 \mathrm{~B}$ configuration, which records visibilities from baselines $214 \mathrm{~m}$ to $6 \mathrm{~km}$. A total bandwidth of $128 \mathrm{MHz}$ split in 32 channels was used for each frequency. The absolute flux density scale was determined using PKS B1934-638 as the primary amplitude and bandpass calibrator (assuming $S_{6 \mathrm{~cm}}=5.83 \mathrm{Jy}$ and $S_{3.6 \mathrm{~cm}}=2.84 \mathrm{Jy}$ ). Periodic observations of PKS B1729-37 were used to correct for changes in gain and phase caused by receiver, local oscillator and atmospheric instabilities. Image processing at all frequencies was carried out under MIRIAD software package (Sault \& Killeen 1999).

To improve the uv coverage, the $\lambda 6 \mathrm{~cm}$ ATCA observations were combined in the uv plane with VLA archive data acquired in the same radio band with the interferometer operating in the hybrid DnC configuration (program AB254 observed July 19 and 22 1984, PI Becker). Finally, single dish data at $\lambda 6 \mathrm{~cm}$ acquired with the Parkes $64 \mathrm{~m}$ telescope (extracted from Parkes-MIT-NRAO Southern Survey, Condon et al. 1991) were combined with the interferometric data using the MIRIAD task IMMERGE, that linearly merges together two images with different resolutions after appropriately weighting both data sets according to their respective primary beam shapes. This addition allowed us to recover all missing flux density due to the lack of short spatial frequencies. The faint structures associated with the outer SNR shell are, however, barely detectable because, in addition to their intrinsic faintness, they suffer from attenuation produced near the primary beam edge. The astrometry in the final image was checked with the $6 \mathrm{~cm}$ Catalog of Compact Radio Sources in the Galactic Plane (White et al. 2005) which has rms positional errors lower than 0.'67 and 0.'84 in RA and DEC, respectively. The resulting synthesized beam and rms noise in the $\lambda 6 \mathrm{~cm}$ image are listed in Table 1 together with the observational parameters for the other analyzed wavelengths. It is worthwhile to note that the new $\lambda 6 \mathrm{~cm}$ image of G0.9+0.1 improves in over 
Table 1. Observational parameters of the radio data.

\begin{tabular}{clll}
\hline \hline $\begin{array}{c}\lambda \\
(\mathrm{cm})\end{array}$ & $\begin{array}{l}\text { Instrument } \\
\text { and configuration }\end{array}$ & $\begin{array}{l}\text { Beam } \\
\left({ }^{\prime \prime} \times^{\prime \prime}\right)\end{array}$ & $\begin{array}{l}\text { Noise } \\
(\mathrm{mJy} / \mathrm{b})\end{array}$ \\
\hline 3.6 & ATCA (6B), VLA (D) & $1.5 \times 0.8$ & 0.05 \\
6 & ATCA (6B), VLA (DnC),SD & $2.9 \times 1.6$ & 0.07 \\
20 & VLA (A, CnB) & $2.5 \times 1.2$ & 0.12 \\
\hline
\end{tabular}

Table 2. Summary of the XMM-Newton observations used in this paper.

\begin{tabular}{cccc}
\hline \hline Obs-Id & Instrument & Time & $\begin{array}{c}\text { Time after } \\
\text { flare screening }\end{array}$ \\
\hline 0112970201 & M1 & $17.2 \mathrm{ks}$ & $16.5 \mathrm{ks}$ \\
0112970201 & M2 & $17.2 \mathrm{ks}$ & $16.2 \mathrm{ks}$ \\
0112970201 & PN & $11.8 \mathrm{ks}$ & $9.3 \mathrm{ks}$ \\
0144220101 & M1 & $49.4 \mathrm{ks}$ & $25.3 \mathrm{ks}$ \\
0144220101 & M2 & $49.4 \mathrm{ks}$ & $29.0 \mathrm{ks}$ \\
0144220101 & PN & $43.7 \mathrm{ks}$ & $15.7 \mathrm{ks}$ \\
\hline
\end{tabular}

50 times the noise level with respect to the previously published image at the same frequency.

To produce a high-fidelity image at $\lambda 3.6 \mathrm{~cm}$, we searched for more databases at this frequency to improve the uv coverage. The ATCA data were combined with VLA D-array archive data obtained at the same frequency (observed on March 25, 2003, program AJ302, PI Rupen), after applying an appropriate calibration factor. This image is only useful to investigate the central PWN since the surrounding radio shell (with a size of $\sim 8^{\prime}$ ) exceeds the primary beam size of the used telescopes at this wavelength $\left(\sim 5^{\prime}\right)$.

A new $\lambda 20 \mathrm{~cm}$ image was produced from the combination of archival VLA A-configuration data (observed in July 3, 1991, program AF209, PI Frail) with data obtained in the VLA hybrid BnC configuration (observed in February 6 and 7 2004, program AY147, PI Yusef-Zadeh). The A-array observations were carried out at 1465 and $1515 \mathrm{MHz}$, using 1331+305 and 1751-253 as primary and secondary calibrators, respectively. The CnB observations were done at 1385 and $1465 \mathrm{MHz}$. In this case $1328+307$ and 1748-253 were used as primary flux density and secondary phase calibrator, respectively. Because of the different observing conditions, both databases were separately calibrated and cleaned and later combined using the task IMMERGE within MIRIAD software, after deciding appropriate overlapping ring in the uv plane. In spite of the special care put in the cleaning process to mitigate the effects of the strong neighbour source Sgr B2 within the observed field, some residual striation could not be completely removed.

\subsection{X-ray band}

We have analyzed the two existing sets of XMM-Newton data on the PWN G0.9+0.1. The first observation, performed in September 2000, was published in Porquet et al. (2003). A second longer observation was carried out in March 2003. The medium filter was set for all cameras (MOS and PN) in the two observations. The data were processed using the Science Analysis System (SAS version 7.1). The periods of high particle background (associated with flares) were rejected. Table 2 summarizes the available observations and exposure time for each of the EPIC cameras, before and after flare screening. Despite the loss of a significant fraction of the observing time in the second observation, the statistics is improved compared to our previous

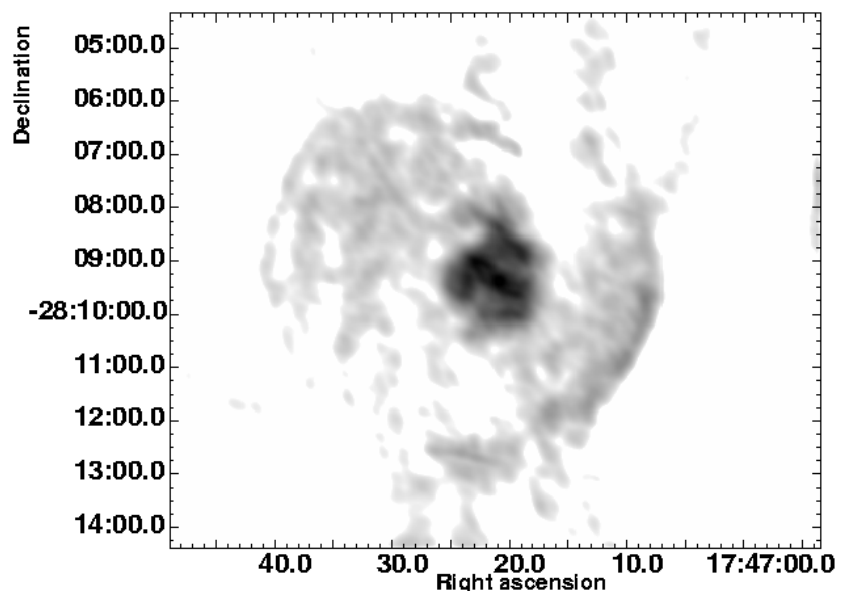

Fig. 2. The SNR G0.9+0.1 at $\lambda 20 \mathrm{~cm}$. The angular resolution is $133^{\prime \prime} 1 \times 88^{\prime \prime} 6$ and the rms noise of $0.25 \mathrm{mJy}$ beam $^{-1}$.

analysis (Porquet et al. 2003), allowing a better comparison with the new high spatial resolution radio data.

We also reprocessed archival Chandra data, originally published by Gaensler et al. (2001), to produce an X-ray image of the PWN at higher spatial resolution. The observation (Obs$\mathrm{id}=1036$, Seq-num $=500$ 102) was performed in October 2000 for an exposure time of $35 \mathrm{ks}$. The image of the PWN was produced in the 3-8 keV energy band. The tool SMOOTH from the SAS was used to provide an adaptively smoothed image at a signal to noise of 10 .

\section{Radio results}

Figure 2 shows the new radio image of the SNR G0.9+0.1 obtained at $\lambda 20 \mathrm{~cm}$ based only on the VLA CnB observations. The features are similar to those observed at $\lambda 90 \mathrm{~cm}$ (Fig. 1), with the brightest side of the surrounding shell to the west and indication of multiple faint arcs in the eastern half. This new sensitive image of G0.9+0.1 shows considerable diffuse emission in the interior of the SNR.

\subsection{Morphology of the PWN}

In Fig. 3 we display the new $\lambda 6 \mathrm{~cm}$ image of the PWN in G0.9+0.1. Figure 4 includes the 3.6, 6 and $20 \mathrm{~cm}$ images of the PWN together with the $90 \mathrm{~cm}$ image taken from Nord et al. (2004) for comparison.

One of the major contributions of this work is the highfidelity representation attained in the 3.6 and $6 \mathrm{~cm}$ images, which have revealed that the radio synchrotron emission from the confined wind in G0.9+0.1 has a complex morphology with multiple small and large scale features, including enhancements along filaments, bright knots and holes. The various conspicuous features (individualized in the image at $\lambda 6 \mathrm{~cm}$ in Fig. 4) are present at all frequencies over the wide spectral range between $330 \mathrm{MHz}$ $(90 \mathrm{~cm})$ and $8.6 \mathrm{GHz}(3.6 \mathrm{~cm})$, although the respective brightness vary across the spectrum.

The morphology is dominated by a central band that runs approximately from NE to SW mimicking at a larger scale the "torus" feature noticed in the Chandra image by Gaensler et al. (2001). This band, that appears defining a symmetry axis, has at least two main maxima (M1 and M2 in Fig. 4), the brightest of which is the westernmost peak, M1. From the image at 


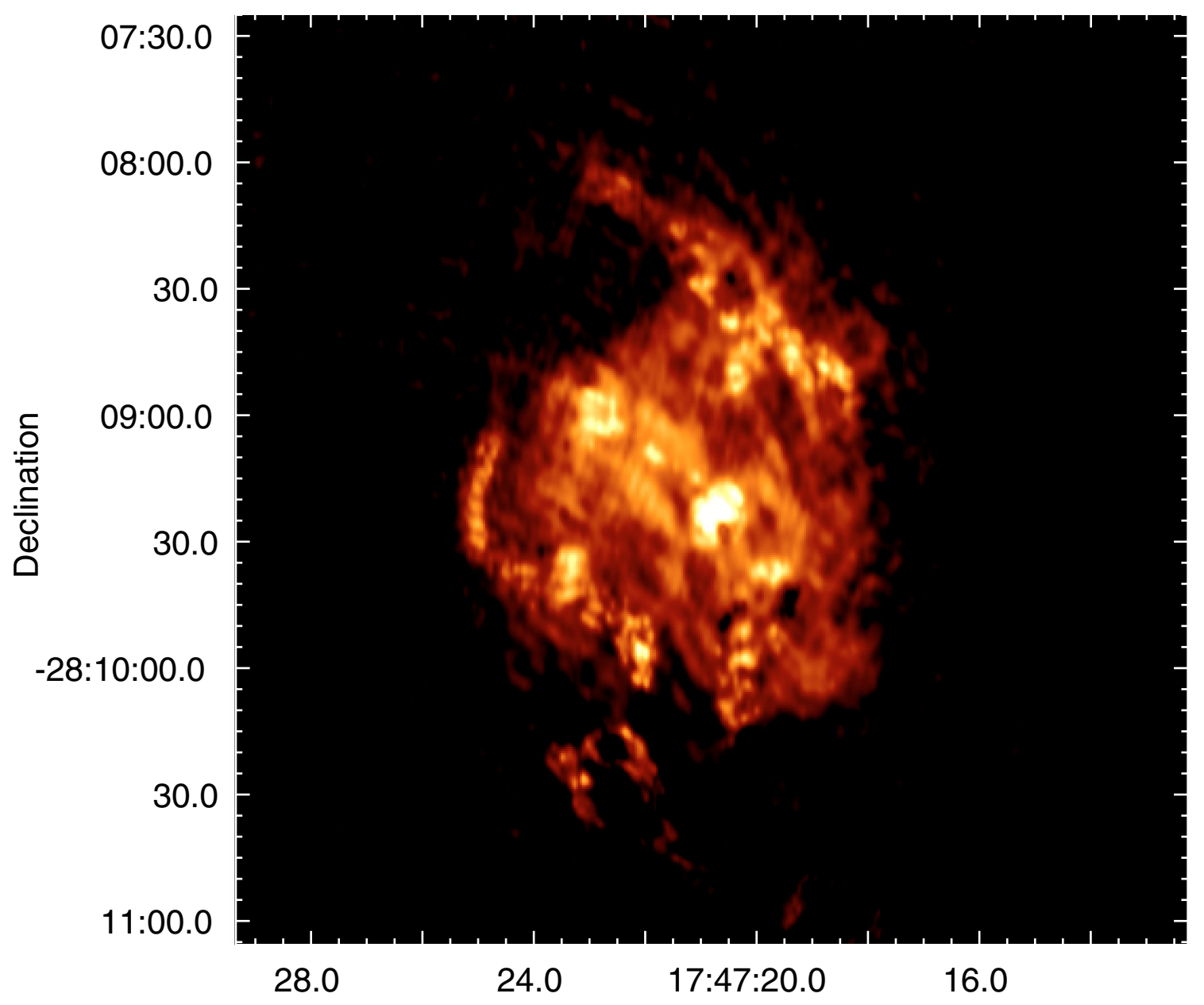

Right ascension

Fig. 3. Color image of the central PWN in the SNR G0.9+0.1 at $\lambda 6 \mathrm{~cm}$. The angular resolution is $2.9 \times 11^{\prime \prime} 6$ and the rms noise $0.07 \mathrm{mJy} / \mathrm{b}$.

$\lambda 6 \mathrm{~cm}$, it looks as if this maximum is the result of the overlap of two unresolved concentrations, of which only one (centered near $\left.17^{\mathrm{h}} 47^{\mathrm{m}} 22^{\mathrm{s}} .8,-28^{\circ} 09^{\prime} 00^{\prime \prime}\right)$ remains visible at $\lambda 3 \mathrm{~cm}$. The maximum M2, centered at $17^{\mathrm{h}} 47^{\mathrm{m}} 22^{\mathrm{s}} .8,-28^{\circ} 09^{\prime} 00^{\prime \prime}$, about $15^{\prime \prime}$ north of the location of CXOU J174722.8-280915 is resolved at $\lambda 6$ and $3.6 \mathrm{~cm}$, revealing that it is not compact, but hollow in the center.

Another interesting feature is the clumpy maximum M3, to the south of the nebula. This maximum is apparently the termination of an almost vertical filament that links M2 with M3 (more clearly seen in the images at $\lambda 90$ and $20 \mathrm{~cm}$ ) and will be discussed below in connection with the X-ray emission. At the periphery of the wind nebula, four striking features can be recognized. The northern border terminates in a curious filament pointing to the east (named Filament 1 in Fig. 4), while the southern limb also has a similar narrow filament on the eastern corner, but in this case the extension points to the north along constant $\mathrm{RA} \sim 17^{\mathrm{h}} 47^{\mathrm{m}} 25^{\mathrm{s}}$ (Filament 2 ). In addition, near the north-western corner a curious small synchrotron circular ring is present (Ring 1 in the $\lambda 6 \mathrm{~cm}$ image, centered near $17^{\mathrm{h}} 47^{\mathrm{m}} 18^{\mathrm{s}}$, $\left.-28^{\circ} 08^{\prime} 40^{\prime \prime}\right)$ and close to the southern border of the nebula but detached from it, a set of short filaments can be observed around $\sim 17^{\mathrm{h}} 47^{\mathrm{m}} 23^{\mathrm{s}},-28^{\circ} 10^{\prime} 30^{\prime \prime}$ forming another incomplete ring-like feature (Ring 2). The appearance of Filaments 1 and 2, protruding from the PWN, resemble the filaments observed in the Crab
Nebula (Hester et al. 1996), and are likely to be originated in magnetic Rayleigh-Taylor instabilities at the interface between the expanding PWN and its surrounding SNR.

Our sensitive new images do not reveal any point source that could be interpreted as the radio counterpart of CXOU J174722.8-280915, the faint hard X-ray source proposed as the best candidate for a central pulsar (indicated by a black cross in Fig. 4). This is not unexpected however, since a typical young radio pulsar distant $\sim 8.5 \mathrm{kpc}$ with an assumed luminosity $L_{1.4 \mathrm{GHz}} \sim 56 \mathrm{mJy} \mathrm{kpc}^{2}$ (like the median for "high-luminosity" young rotation-powered pulsars as estimated by Camilo et al. 2006), would have a flux density $S_{1.4 \mathrm{GHz}} \sim 0.08 \mathrm{mJy}$, well below the sensitivity of the $1.4 \mathrm{GHz}$ image. Moreover, to be detectable such a point-like source should be brighter than the $\sim 70 \mathrm{~mJ} /$ beam nebular emission in its vicinity.

\subsection{Flux density measurements and spectral study}

In the early paper reporting the discovery of G0.9+0.1, Helfand \& Becker (1987) estimated the flux density of the PWN (called "the core" in their paper) at $\lambda 6$ and $20 \mathrm{~cm}$ on the basis of VLA observations. The calculations were carried out by integrating the detected emission at both wavelengths within a square box $2^{\prime}$ on a side and, under some assumptions, the authors derive $S_{\text {core- } 6 \mathrm{~cm}}=4.16 \mathrm{Jy}$ and $S_{\text {core-20 } \mathrm{cm}}=4.15 \mathrm{Jy}$. For the shell 


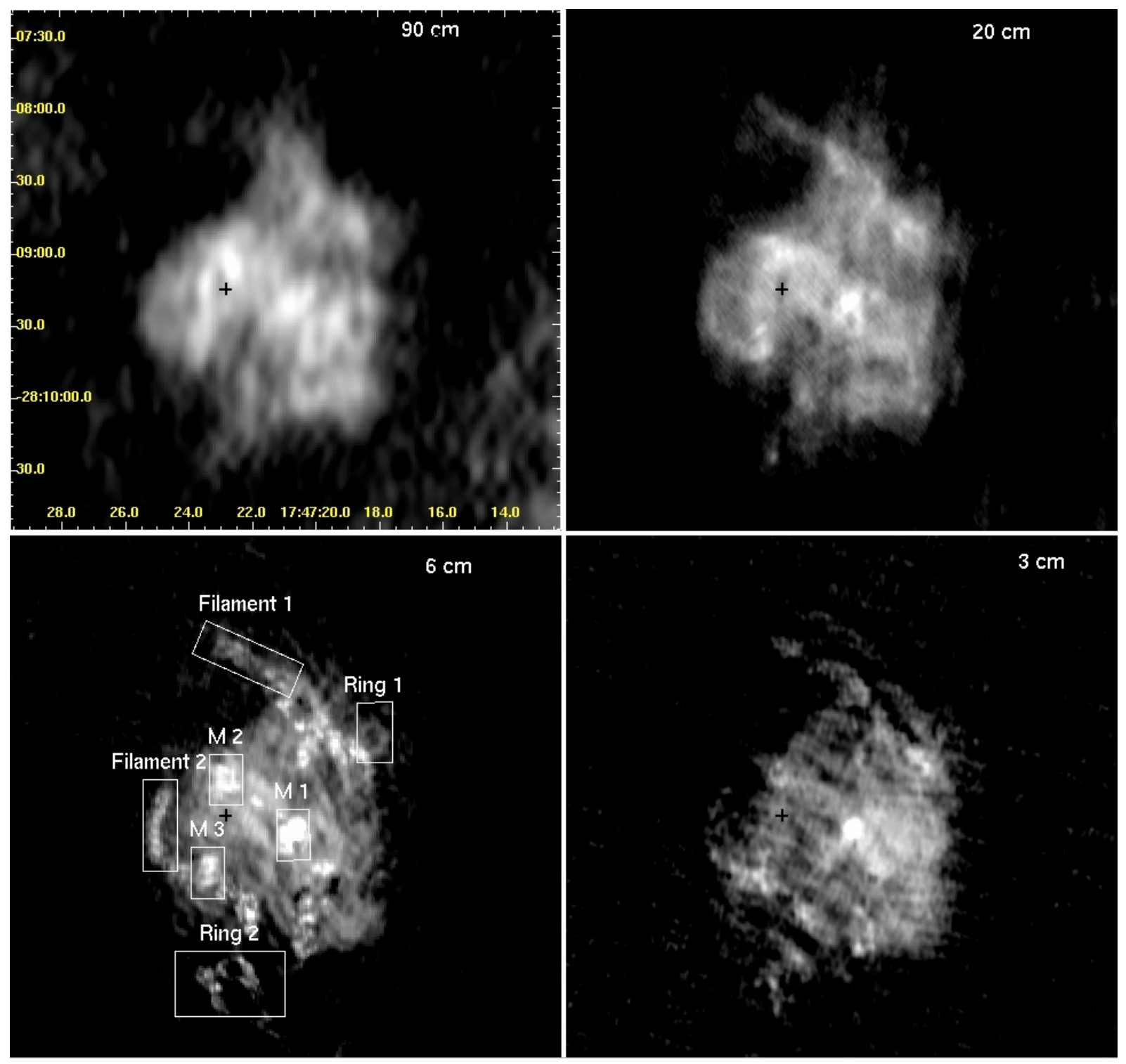

Fig. 4. Grey-scale images of the central PWN in the SNR G0.9+0.1 at 90, 20, 6 and $3.6 \mathrm{~cm}$ wavelengths. The black cross marks the position of the X-ray point source CXOU J174722.8-280915. The different features individualized in the panel corresponding to the $6 \mathrm{~cm}$ image are discussed in the text.

emission, at $6 \mathrm{~cm}$ the observational limitations (antennas shadowing limiting the VLA sensitivity for features on the largest angular scales) are compensated by crudely adding a zero-spacing flux density to the uv data before mapping. In this way the authors derive $S_{\text {shell-6 }} \mathrm{cm} \sim 7.9 \mathrm{Jy}$. For the estimates at $\lambda 20 \mathrm{~cm}$, the authors note that it is not possible to apply the same method than before because of the presence of the bright source Sgr B2 at the edge of the observed field, deriving under some considerations $S_{\text {shell-20 cm }} \sim 14.4 \mathrm{Jy}$. Finally, these results were combined with data collected from the literature between $408 \mathrm{MHz}$ and $22 \mathrm{GHz}$ to carry out a global spectral study. Here the authors reasonably note that because of the different beams used in the various multifrequency observations, it is apparent that some observers measured only the core, i.e. the PWN alone, while others included some or all of the shell emission. From this combination, Helfand \& Becker (1987) obtain $\alpha_{\text {core }} \sim-0.12$ and $\alpha_{\text {shell }} \sim-0.6$, concluding that G0.9+0.1 is a composite SNR.

Later La Rosa et al. (2000) observed G0.9+0.1 at $\lambda 90 \mathrm{~cm}$ as a part of the Galactic Center study using the VLA $(H P B W \sim$ $43^{\prime \prime}$ and rms sensitivity $0.5 \mathrm{mJybeam}^{-1}$ ). In this work the authors report $S_{\text {shell-90 } \mathrm{cm}} \sim 16 \mathrm{Jy}$ and $S_{\text {core-90 } \mathrm{cm}} \sim 3.5 \mathrm{Jy}^{3}$ concluding, after comparing with $20 \mathrm{~cm}$ VLA data, that $\alpha_{\text {core }} \sim$ +0.12 and $\alpha_{\text {shell }} \sim-0.77$, which confirms the classification as a composite remnant suggested by Helfand \& Becker (1987).

Although it is beyond any doubt that the SNR G0.9+0.1 has two different spectral components, the PWN with a flat spectrum and the shell with steeper spectrum, it is useful to revise the flux density estimates based on the new observations where the contributions at all spatial scales have been adequately considered and processed using modern image reconstruction algorithms. Besides, the new images with very good spatial resolution allow us to accurately determine the areas considered to spatially integrate the flux density; also source brightness and background contributions are now estimated on the basis of very sensitive data.

\footnotetext{
3 Note that in Table 1 the authors list a flux density of $4.8 \mathrm{Jy}$ for the core, while in the text the value mentioned for the same component is 3.5 Jy, after correcting for diffuse background.
} 
Table 3. Integrated radio flux densities.

\begin{tabular}{ccccc}
\hline \hline $\begin{array}{c}\lambda \\
(\mathrm{cm})\end{array}$ & $\begin{array}{c}S_{\text {SNR }} \\
(\mathrm{Jy})\end{array}$ & $\begin{array}{c}S_{\mathrm{PWN}} \\
(\mathrm{Jy})\end{array}$ & $\begin{array}{c}S_{\mathrm{PWN}}^{\text {corr }} \\
(\mathrm{Jy})\end{array}$ & $\begin{array}{c}S_{\text {shell }} \\
(\mathrm{Jy})\end{array}$ \\
\hline 3.6 & & $1.57 \pm 0.40$ & $1.35 \pm 0.50$ & \\
6 & $\geq 6.90 \pm 0.20$ & $3.20 \pm 0.14$ & $1.45 \pm 0.23$ & $\geq 3.70 \pm 0.25$ \\
20 & $11.50 \pm 0.70$ & $3.23 \pm 0.20$ & $1.72 \pm 0.30$ & $8.27 \pm 0.70$ \\
\hline
\end{tabular}

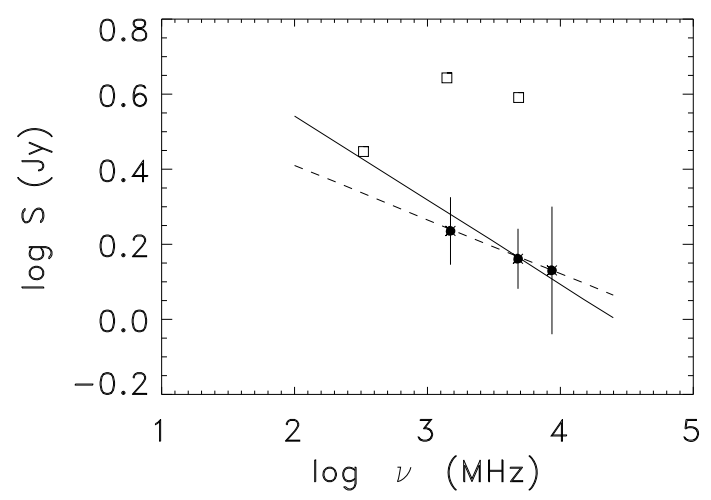

Fig. 5. Radio spectrum of the PWN in G0.9+0.1. The data from this work are shown by filled circles; data at 1400 and $4850 \mathrm{MHz}$ from Helfand \& Becker (1987) and data at $330 \mathrm{MHz}$ from La Rosa et al. (2000) are displayed as open squares. The best linear fit based on the new data plus the $330 \mathrm{MHz}$ (solid line) produces $\alpha=-0.22$. A linear fit to the new data alone (dashed line) produces $\alpha=-0.14$. Data from Helfand \& Becker (1987) were not used in the fitting. An average $\alpha=-0.18 \pm 0.04$ is adopted.

In Table 3 we list the flux densities estimated from the new 3.6, 6 and $20 \mathrm{~cm}$ images. The first column lists the total SNR emission (that is, including contributions from the external arcs, diffuse interior and core), the second column corresponds to the core component alone, the third one has the same but with the underlying shell emission subtracted, and the fourth one lists the shell component alone. The errors quoted in Table 3 include the intrinsic noise, as well as possible uncertainties introduced in the selection of the level of background contribution.

At $\lambda 3.6 \mathrm{~cm}$, only the flux density of the core component is listed because, as mentioned in Sect. 2.1, at this wavelength the shell cannot be appropriately mapped with a single pointing. At $\lambda 6 \mathrm{~cm}$, the presence of diffuse emission associated with the Galactic plane contaminates the SNR flux density estimates. This effect was considered by subtracting a background at a level that leaves the SNR G0.9+0.1 emission clearly detached from surrounding emission. Also, only lower limit is listed for the flux density at $\lambda 6 \mathrm{~cm}$ of the whole SNR since, as mentioned in Sect. 2.1, at this wavelength the outer shell contribution is probably not completely recovered. Though Table 3 lists for completeness the core flux density before correcting for diffuse shell emission contribution, all subsequent calculations are carried out based on the corrected flux density.

Figure 5 shows the global spectrum of the central PWN in G0.9+0.1 between $330 \mathrm{MHz}$ and $8400 \mathrm{MHz}$ together with the least square fits to our data alone (dashed line) and to our data plus La Rosa et al. 2000's $330 \mathrm{MHz}$ data (solid line). The flux densities published by Helfand \& Becker (1987) were not taken into account in this new fitting since, for the reasons mentioned above, they can be overestimated. In the case of including the $90 \mathrm{~cm}$ data, the PWN spectral index is $\alpha_{\mathrm{PWN}}=-0.22$, while based on 20, 6 and $3.6 \mathrm{~cm}$ data alone, $\alpha_{\mathrm{PWN}}=-0.14$. In what follows we adopt for the PWN $\alpha_{\text {PWN }}=-0.18 \pm 0.04$.

For the shell component we have compared the $90 \mathrm{~cm}$ image with the new $20 \mathrm{~cm}$ image, obtaining $\alpha_{\text {shell }}=-0.68 \pm 0.07$, in agreement with previous estimates.

The study of spatial variations of the spectrum across the PWN is a sensitive tool for understanding the coupling between the fresh relativistic electrons and magnetic fields constantly supplied by the pulsar and the surrounding plasma. Therefore, based on the good quality images obtained at three frequencies, we followed different procedures to investigate possible spatial spectral variations across the PWN.

First, the spectral study was carried out by performing the direct comparison of the different images. To assure that the range of spatial scales measured at each frequency was perfectly matched, we applied an appropriate uv tapering and reconstructed the interferometric images. In addition, to avoid positional offsets, the images were aligned and interpolated to identical projections (field center and pixel size). We repeated the ratio of the images, first at the angular resolution of the considered images and second, after degrading the spatial resolution to $5^{\prime \prime} \times 5^{\prime \prime}$ and to $10^{\prime \prime} \times 10^{\prime \prime}$ in order to minimize possible bias, like small scale image artifacts, zero level differences, background variations, etc., that could mask real variations. Contrary to what was shown in the X-rays domain by Porquet et al. (2003) where the eastern side of the PWN was found to have a clearly flatter (and harder) spectrum than the southwestern half, we obtained that the radio spectral index distribution is practically featureless, with no particular morphology/spectrum correspondence or tendency.

To investigate if the lack of conspicuous spectral variations originated in the procedure, we repeated the study using "tomographic" images (see for example Katz-Stone \& Rudnick 1997), a method where different spectral indices are tested and residuals with a spectrum flatter (or steeper) than the $\alpha_{\text {test }}$ are highlighted as darker (or lighter) features in an otherwise uniform grey map. This method is very sensitive to fine-scale spectral index changes. Again the result was that no clear departures from a mean spectral index of $\alpha \sim-0.2$ are evident within the PWN. To accurately trace small local variations in the spectral index, a set of homogeneous observations acquired at different wavelengths using the same instrument and with analogous observing conditions, would be necessary.

\section{Radio/X-ray comparison}

The improved X-ray images of G0.9+0.1 were used to compare with the radio brightness distribution. The full comparison with X-ray images, however, lacks either better spatial resolution ( 8 " for XMM-Newton) or higher statistics (only $35 \mathrm{ks}$ of Chandra data).

Figure 6 shows the new 3-6 keV XMM-Newton image (left panel) and the 3-8 keV Chandra image (right panel) compared with the radio image obtained at $6 \mathrm{~cm}$ (central panel to facilitate visual comparison with both $\mathrm{X}$-ray datasets).

It is significant the fact that the maxima in the two spectral regimes do not coincide. The X-ray peak is approximately $15^{\prime \prime}$ southern of the radio maximum M2, while at the position of the brightest radio maximum, M1, only weak X-ray emission is detected.

The improved X-ray images confirm that X-rays and radio emitting areas have a comparable extent. Good radio/X-ray correspondence can be noticed between the termination of the "jetlike" feature noticed by Gaensler et al. (2001) in an approximate 


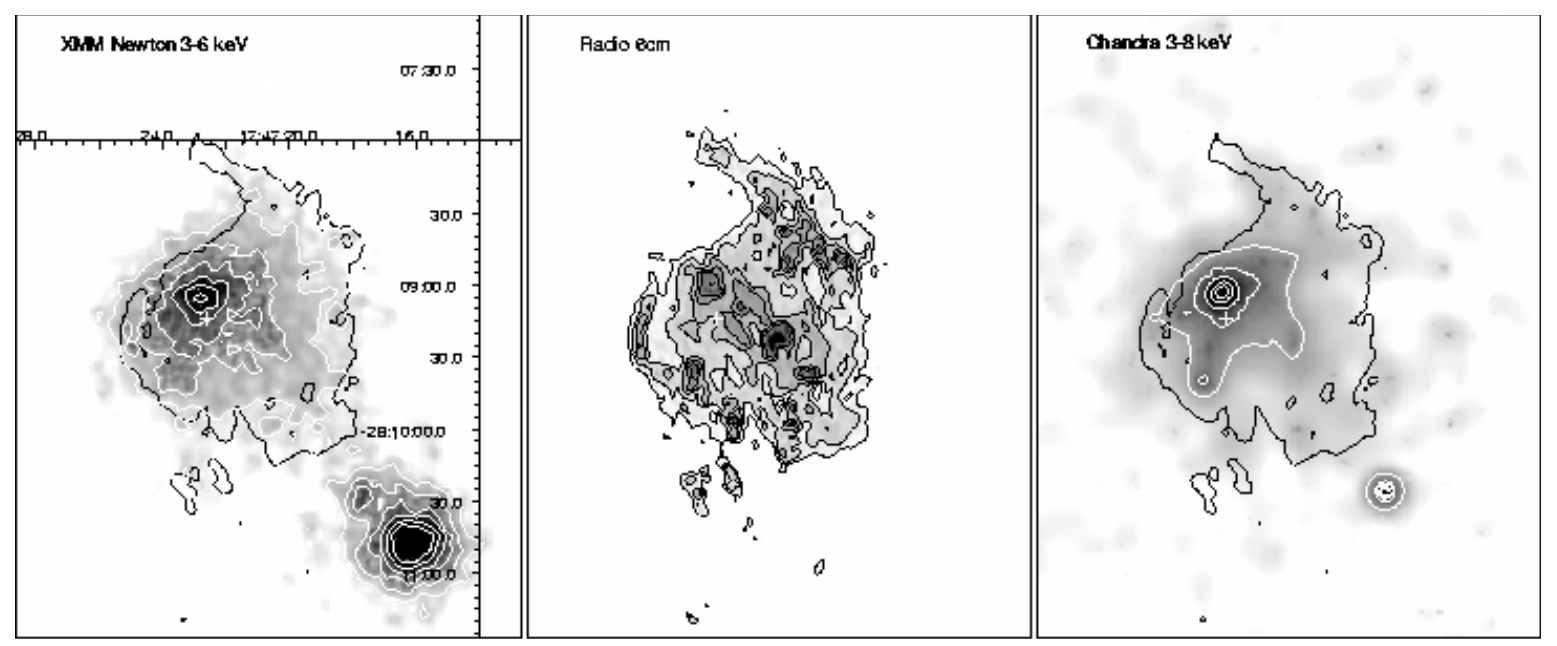

Fig. 6. Reprocessed XMM-Newton and Chandra X-ray images displayed together with the $6 \mathrm{~cm}$ radio image. Both X-ray images have X-ray contours (white) and a radio contour (black) overlapped to facilitate the identification of features and the multispectral comparison. The location of the pulsar candidate CXOU J174722.8-280915 is marked by a white plus sign.

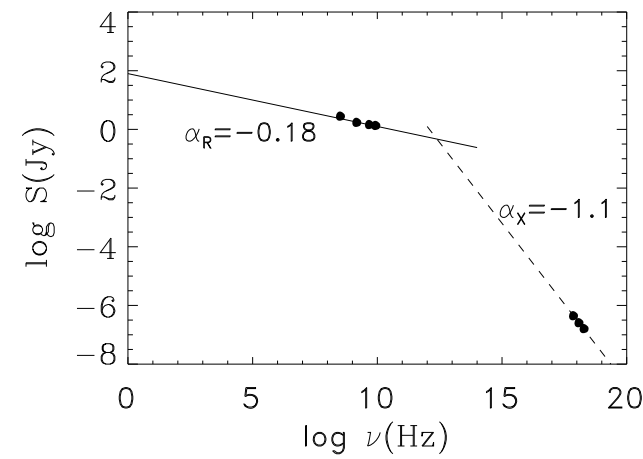

Fig. 7. Spectrum of the PWN in G0.9+0.1 over the range $10^{8}$ to $10^{19} \mathrm{~Hz}$, with a spectral index $\alpha_{\mathrm{R}}=-0.18$ in the radio regime and $\alpha_{\mathrm{X}}=-1.1$ as derived from X-ray data. The extrapolation of the X-ray spectrum intersects that of the radio spectrum at the turnover frequency $v_{\mathrm{b}}=2.4 \times$ $10^{12} \mathrm{~Hz}$.

north-south direction and interpreted as a jet directed along the pulsar spin axis, and the radio maximum M3 located exactly at the southern extreme of the X-ray feature. Besides, as mentioned in Sect. 3.1, at $\lambda 90 \mathrm{~cm}$ and $\lambda 20 \mathrm{~cm}$, the radio emission strikingly follows the shape and extension of the entire X-ray jet.

Based on the XMM-Newton data we have estimated the $X$-ray flux in: $2.14 \times 10^{12} \mathrm{erg} \mathrm{cm}^{-2} \mathrm{~s}^{-1}$ between 2 and $4 \mathrm{keV}$, $1.24 \times 10^{12} \mathrm{erg} \mathrm{cm}^{-2} \mathrm{~s}^{-1}$ between 4 and $6 \mathrm{keV}$, and $1.56 \times$ $10^{12} \mathrm{erg} \mathrm{cm}^{-2} \mathrm{~s}^{-1}$ in the 6 to $10 \mathrm{keV}$ interval. Following the procedure used for the radio data, we applied a least square fit to the X-ray data, obtaining a spectral index $\alpha_{\mathrm{X}}=-1$.1, which corresponds to a photon index $\Gamma=1-\alpha=2.1$, which equals the average value between the hardest spectrum derived by Porquet et al. (2003) on the eastern side of the PWN $(\Gamma=1.0)$ and the softest one $(\Gamma=3.2)$ on the western part. In Fig. 7 we display the radio synchrotron spectrum shown in Fig. 5 together with the X-ray data. From the intersection of the spectral fitting in radio and in the $\mathrm{X}$-ray domain, we can conclude that the spectrum must break around $v_{\mathrm{b}}=2.4 \times 10^{12} \mathrm{~Hz}$.

\section{Energetics of the PWN}

Based on the estimated radio flux density, break frequency $v_{\mathrm{b}}$ and radio spectral index, we can calculate the radio luminosity associated with the PWN in G0.9+0.1 between $\sim 10^{7} \mathrm{~Hz}$ and $v_{\mathrm{b}}$. A total $L_{\text {radio }} \sim 1.2 \times 10^{35} \mathrm{erg} \mathrm{s}^{-1}$, is obtained. This radio luminosity can be compared to $L_{\mathrm{X}} \sim 4.7 \times 10^{34} \mathrm{erg} \mathrm{s}^{-1}$ measured by Porquet et al. (2003) between 2 and $10 \mathrm{keV}$, after correcting for $d=8.5 \mathrm{kpc}$. The luminosities ratio, a useful parameter because it is independent of the distance, turns out to be $L_{\mathrm{X}} / L_{\text {radio }} \sim 0.4$.

It is of great interest to determine the energy requirements for the radio synchrotron emission associated with the PWN. Energy is stored in the particles as well as in the magnetic field. Following Moffet (1975) we can express the total energy of the source as

$$
U_{\mathrm{T}}=U_{\mathrm{p}}+U_{\mathrm{m}}=a A L B^{-3 / 2}+V B^{2} / 8 \pi
$$

where $L$ is the total radio luminosity of the nebula, $V$ its volume, $a$ represents the ratio between the energy in relativistic electrons and in energetic baryons, and is assumed to be 1 , while $A$ depends on the lower and upper cutoff frequencies and can be estimated as follows:

$A=\frac{C_{1}{ }^{1 / 2}}{C_{3}} \frac{(2 \alpha+2)}{(2 \alpha+1)} \frac{\left(v_{1}{ }^{\alpha+1 / 2}-v_{0}{ }^{\alpha+1 / 2}\right)}{\left(v_{1}{ }^{\alpha+1}-v_{0}{ }^{\alpha+1}\right)}$.

The total energy as a function of the magnetic field $B$ attains its minimum when the magnetic energy is approximately equal to the particle energy (equipartition situation). It is usually assumed that the magnetic field in the source is actually equal to the value which gives minimum energy. The minimum total energy is $U_{\min }=0.50(a A L)^{4 / 7} V^{3 / 7}=7 \times 10^{47} \mathrm{erg}$ and the magnetic field for which equipartition holds can be calculated from: $B\left(U_{\min }\right)=2.3(a A L / V)^{2 / 7}=56 \mu \mathrm{G}$. This value agrees with the magnetic field strength early proposed by Helfand \& Becker (1987) for this PWN (though they assumed a higher $v_{\mathrm{b}}$ ), and is comparable to the value estimated for the PWN in the SNR W44 (Petre et al. 2002). Aharonian et al. (2005) estimated a magnetic field strength of only $6 \mu \mathrm{G}$ for the PWN in G0.9+0.1, though this number is inferred from model fitting to the broad-band spectral energy distribution, where the $B$ field is a free parameter.

Based on our calculated magnetic field, the magnetic energy is $U_{\mathrm{m}} \sim 2 \times 10^{47} \mathrm{erg}$ and the particle energy $U_{\mathrm{p}} \sim 5 \times 10^{47} \mathrm{erg}$. The wind magnetization parameter (ratio between magnetic flux energy density to that in particles) is then $\sigma \sim 0.4$. Such a high value suggests that the wind just started to become particle dominated, though the transition from $\sigma>1$ to $\sigma<1$ is not yet clear (see Arons 1998, for discussion). 


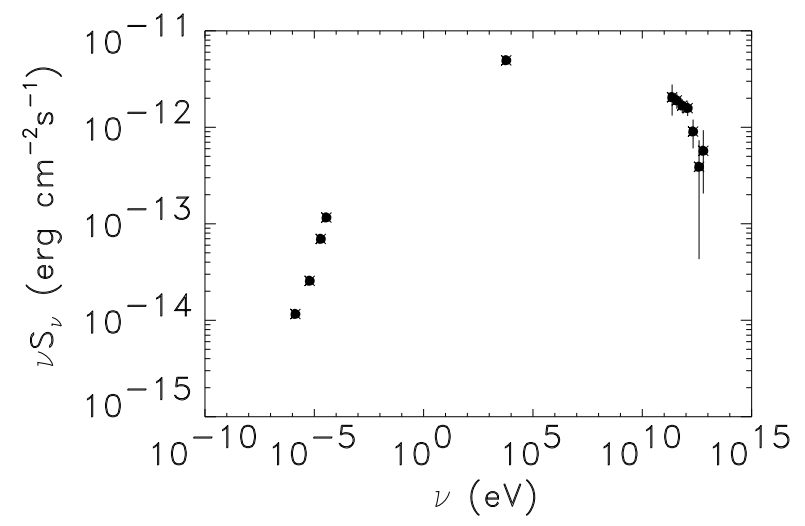

Fig. 8. Spectral energy distribution of the PWN in G0.9+0.1 from radio to VHE gamma-ray, joining the new estimates in the radio and X-ray bands with HESS measurements.

A rough estimate of the age of the PWN can be estimated from the comparison of the total energy with the rate of rotational energy loss of the pulsar. This last parameter can be derived from the empirical relation between the X-ray luminosity and E proposed by Possenti et al. (2002):

$\log L_{\mathrm{X}}=1.34 \log \dot{E}-15.3$.

From this relation, an energy loss rate $\dot{E} \sim 2 \times 10^{37} \mathrm{erg} \mathrm{s}^{-1}$ is derived, thus implying that the pulsar had to be continuously injecting energy during, at least, $\sim 1100 \mathrm{yr}$ to create the observed PWN.

As a by-product of the new radio measurements, in Fig. 8 we show the broadband spectral energy distribution (SED) of the PWN in G0.9+0.1 obtained after combining the radio data presented in this paper plus the $330 \mathrm{MHz}$ data from La Rosa et al. (2000) together with the X-ray data and VHE HESS measurements (from the public HESS database).

\section{Conclusions}

This paper presents new high-resolution and high-sensitivity images of the PWN in the SNR G0.9+0.1 obtained at different radio frequencies. The study is complemented with reprocessed X-ray images based on XMM-Newton and Chandra data. The new radio images have revealed interesting structures in the nebula, like bright knots, rings and elongated filaments which might be showing instability regions at the sites where the expanding nebula interacts with the surrounding ejecta. From the comparison of the radio images with the reprocessed X-ray images it is found that the X-ray emitting electrons largely fills the volume delineated by the radio PWN. Also, the new detailed radio images have confirmed the symmetry suggested by the Chandra $\mathrm{X}$-ray observations, with a bright central band aligned with the X-ray "toroidal" feature and a narrow elongated north-south structure that appears as the counterpart of the "jet-like" X-ray feature. These good radio/X-ray correspondences are, however, accompanied by notable disagreements, the most important of which is the separation observed between the radio and the X-ray maxima.

Based on the new radio images, with contributions from all spatial scales adequately recovered, we estimated the multispectral flux densities and performed a spectral study. In Table 4 we
Table 4. Characteristic parameters of the PWN in G0.9+0.1.

\begin{aligned} \hline \hline$S_{3.6 \mathrm{~cm}} & =1.35 \pm 0.50 \mathrm{Jy} \\ S_{6 \mathrm{~cm}} & =1.45 \pm 0.23 \mathrm{Jy} \\ S_{20 \mathrm{~cm}} & =1.72 \pm 0.30 \mathrm{Jy} \\ \alpha_{\text {radio }} & =-0.18 \pm 0.04 \\ L_{\text {radio }} & \simeq 1.2 \times 10^{35} \mathrm{erg} \mathrm{s}^{-1} \\ v_{\mathrm{b}} & \simeq 2.4 \times 10^{12} \mathrm{~Hz} \\ B & \simeq 56 \mu \mathrm{G} \\ U_{\text {particles }} & \simeq 5 \times 10^{47} \mathrm{erg} \\ U_{\text {magnetic }} & \simeq 2 \times 10^{47} \mathrm{erg} \\$ age & $\geq 1100 \mathrm{yr}\end{aligned}$

summarize these results together with other observed and derived characteristic parameters of the PWN in G0.9+0.1. Our study revealed a quite uniform distribution of radio spectral index across the nebula, with only small fluctuations around the mean value of $\alpha_{\mathrm{r}}=-0.18$.

From the combination of observations in the radio regime with X-ray data we traced a broadband spectrum which suggests a spectral break at $v_{\mathrm{b}} \approx 2.4 \times 10^{12} \mathrm{~Hz}$. On the basis of this information, together with the observed luminosities and the assumption of equipartition between particles energy and Poynting vector energy, we investigated the energetics and the magnetic field in the nebula.

Acknowledgements. We are very grateful to E. Reynoso and to A. Green who participated in the data acquisition and first stages of this work. We thank the referee, Professor David Helfand, for his useful comments that improved the manuscript. We acknowledge M. Nord for providing us with the $330 \mathrm{MHz}$ image used for this study. This research was carried out within the framework of the ECOS-Sud France-Argentina exchange program. The research has been partially funded by grants CONICET PIP 6433, UBACYT A055/04 and ANPCYT-PICT 03-14018 of Argentina. This work is based on observations done with XMM-Newton, an ESA science mission with instruments and contributions directly funded by ESA Member States and the US (NASA). G. Dubner and E. Giacani are members of the Carrera del Investigador Científico of CONICET, Argentina.

\section{References}

Aharonian, F., Akhperjanian, A. G., Aye, K.-M., et al. 2005, A\&A, 432, L25 Arons, J. 1998, Memorie della Societa Astronomica Italiana, 69, 989 Camilo, F., Ransom, S. M., Gaensler, B. M., et al. 2006, ApJ, 637, 456 Gaensler, B. M., Pivovaroff, M. J., \& Garmire, G. P. 2001, ApJ, 556, L107 Gray, A. D. 1994, Proceedings of the Astronomical Society of Australia, 11, 79 Helfand, D. J., \& Becker, R. H. 1987, ApJ, 314, 203

Helfand, D. J., Gotthelf, E. V., \& Halpern, J. P. 2001, ApJ, 556, 380

Hester, J. J., Stone, J. M., Scowen, P. A., et al. 1996, ApJ, 456, 225 Kaspi, V. M., \& Helfand, D. J. 2002, in ASP Conf. Ser., 3

Katz-Stone, D. M., \& Rudnick, L. 1997, ApJ, 479, 258

La Rosa, T. N., \& Kassim, N. E. 1985, ApJ, 299, L13

La Rosa, T. N., Kassim, N. E., Lazio, T. J. W., \& Hyman, S. D. 2000, AJ, 119, 207

Mereghetti, S., Sidoli, L., \& Israel, G. L. 1998, A\&A, 331, L77

Moffet, A. T. 1975, Strong Nonthermal Radio Emission from Galaxies (Galaxies and the Universe), 211

Nord, M. E., Lazio, T. J. W., Kassim, N. E., et al. 2004, AJ, 128, 1646

Petre, R., Kuntz, K. D., \& Shelton, R. L. 2002, ApJ, 579, 404

Porquet, D., Decourchelle, A., \& Warwick, R. S. 2003, A\&A, 401, 197

Possenti, A., Cerutti, R., Colpi, M., \& Mereghetti, S. 2002, A\&A, 387, 993

Roberts, M. S. E., Tam, C. R., Kaspi, V. M., et al. 2003, ApJ, 588, 992

Sault, B., \& Killeen, N. 1999, Miriad Users Guide,

http://www . atnf. csiro.au/computing/software/miriad

Sidoli, L., Mereghetti, S., Israel, G. L., \& Bocchino, F. 2000, A\&A, 361, 719

Weisskopf, M. C., Hester, J. J., Tennant, A. F., et al. 2000, ApJ, 536, L81

White, R. L., Becker, R. H., \& Helfand, D. J. 2005, AJ, 130, 586 